\title{
La sédimentation des réservoirs des grands barrages hydroélectriques et les aspects particuliers de ce problème en Yougoslavie
}

\section{Sedimentation in the reservoirs of large hydroelectric dams, and the special features of the problem in Yugoslavia.}

\author{
PAR J. MESSINES DU SOURBIER \\ INSPECTEUR GÉNËRAL DES EAUX ET FONÊTS \\ PRÉSTDENT DE LA SECTION \&GLACIOLOGIB 》 A LA SOCIÉTÉ HYDROTECHNIQUE DE FRANCE
}

A propos du voyage effectué par le Groupe de Travail de la Correction des Torrents en Yougoslavie du 29 juillet au 19 août 1956.

Le Groupe de Travail de la Correction des Torrents et de la Lutte contre les Avalanches, fondé à Rome par la F.A.O. en 1952, a tenu sa troisième session en Yougoslavie au cours de l'été 1956. Après un voyage d'études d'une dizaine de jours qui lui permit d'examiner les divers aspects de l'érosion en Serbie, Bosnie-Herzégovine et Dalmatie, il acheva ses travaux à Bled en Slovénie.

Les débats portèrent notamment sur les différentes méthodes de défense des sols, qu'il s'agisse des ouvrages de correction torrentielle, c'est-à-dire de la lutte contre l'érosion linéaire, ou de ceux mis en cuvre pour lutter contre l'érosion généralisée des versants : érosion superfirielle ou en nappes.

Les questions relatives à la conservation et à lutilisation des sols en montagne furent également examinées, mais le sujet principal des discussions fut celui de la sédimentation des réservoirs des grands barrages.

Le rapporteur en était notre collegue, M. Widmann, Conservateur des Faux et Forêts à TouJouse.

La note ci-dessous s'inspire de l'étude de M. Widmann dans l'exposé de quelques considérations générales et traite ensuite des aspects particuliers de ce problème en Yougoslavie.

Le développement de la « houille blanche», et la construction à travers le monde de retenues artificielles des eaux de plus en plus grandes, ont appelé l'attention des pouvoirs publics et des ingénieurs responsables sur le grave problème de la sédimentation de ces retenues. Le comblement de ces réserves d'eaux est plus ou moins rapide, plus ou moins total, mais il se manifeste presque toujours et compromet leur durée.

Les transports de matériaux ou débits solides des rivières torrentielles sont la cause de ces phénomènes. Or, ces débits solides varient selon les conditions du sol, du relief, de la végétation et du climat. Les débits solides spécifiques annuels, ou coefficients de transports spécifiques, s'expriment en $\mathrm{m}^{3}$ par $\mathrm{km}^{2}$ et par an. Ils peuvent varier selon les régions, de 0 à 9.000 en moyenne, et sont toujours plus élevés dans les petits bassins torrentiels à forte pente que dans les grands bassins hydrographiques, ce qui se concoit fort bien. Enfin, les écarts sur les moyennes sont très grands d'une année à l'autre, en fonction des conditions pluviométriques.

En faisant les réserves nécessaires sur la précision de ces mesures, au sujet desquelles G. Labaye [1], G. Braudeau ]2[, M. Bonnin, etc., ont appelé particulièrement l'attention, nous citerons les exemples suivants:

En France [3], les coefficients de transports spécifiques seraient, en moyenne, de l'ordre de 20 a 50 dans le Massif Central, et de 70 dans les Pyrénées. Ce coefficient atteindrait 500 à 800 dans les grands bassins torrentiels des Alpes, 
comme la Durance supérieure ou le Haut Drac. La valeur de ce débit solide ne serait plus que de 147 dans les Alpes calcaires méridionales, pour le Verdon à Quinson. Il varierait entre 500 et 900 pour le Rhône suisse, en diverses localités du Valais.

En Italie, sous le climat aride de l'Apennin Emilien, dans des formations géologiques très affouillables, les débits solides seraient de $5.280 \mathrm{~m}^{3} / \mathrm{km}^{2}$ dans le bassin de l'Enza.

En Afrique du Nord, des mesures précises effectuées dans un petit bassin de réception, celui de l'Oued el Oudja [4], ont donné en 1947-48 un débit solide de 3.100 , et l'année suivante de $10.000 \mathrm{~m}^{3} / \mathrm{km}^{2} / \mathrm{an}$.

Aux U.S.A., les torrents de la région de Los Angeles [5] ont des débits solides spécifiques moyens variant de 400 à 9.600 et les maxima observés ont été de trois à quatre fois supérieurs, tandis qu'un vaste bassin hydrographique, comme celui du Missouri, accuse seulement à l'embouchure, un débit solide de $118 \mathrm{~m}^{3} / \mathrm{km}^{2} / \mathrm{an}$.

En Yougoslavie, sans atteindre les chiffres records de l'Afrique du Nord ou de certaines régions des Etats-Unis d'Amérique, le débit solide de la Morava du Sud, dans le secteur de Gardelica, bassin de 60.000 hectares, atteindrait le chiffre moyen de $6.700 \mathrm{~m}^{3} / \mathrm{km}^{2} / \mathrm{an}$, ce qui est considérable. Celui de la Neretva supérieure, moins important, serait de l'ordre de $1.000 \mathrm{~m}^{3} / \mathrm{km}^{2} / \mathrm{an}$, tandis que les rivières du Karst en Dalmatie ont un débit solide beaucomp plus faible, parfois même insignifiant.

Il est intéressant de connaître la composition de ce débit solide des rivières. Il faut distinguer d'une part le transport des matériaux en suspension et, d'autre part, celui des matériaux charriés sur le fond.

La part des matériaux de charriage varie, en gros, entre 2 et $50 \%$ du transport total, mais le taux usuel oscille généralement entre 5 et $20 \%$.

Voici quelques estimations :

France. - Barrage du Sautet : $30 \%$. Ce serait plutôt un maximum. - Barrage de SerrePoncon : 8 à $12 \%$, selon les auteurs.

Sursse. - Le Rhône à Louèche-les-Bains : $30 \%$.

U.S.A. - Le Missouri : $10 \%$.

D'après nos observations en Yougoslavie, i] semble, par analogies, que le charriage des matériaux sur le fond, par saltation, dans la Morava du Sud, à hauteur du défilé de Gardelica, soit très faible, de l'ordre de $5 \%$ seulement. II est certainement plus élevé, mais ne doit pas dépasser $15 \%$ dans le bassin de la Neretva. Cependant, faute de mesures directes, ces estimations sont données sous toutes réserves.

En ce qui concerne les matériaux transportés en suspension, la difficulté des mesures tient principalement à la variabilité de la répartition de la teneur en sédiments dans le courant liquide suivant la profondeur. On aboutit cependant à des résultats acceptables pour les colloïdes, les argiles fines, le silt fin, permettant d'apprécier d'une manière suffisanle la turbidité des rivières. Mais il est plus délicat d'apprécier la part de ce débit solide en suspension qui se dépose dans les réservoirs des grands barrages, à l'amont de ces derniers. Les matériaux fins en suspension (silt et argile) et les argiles qui ont subi le phénomène de la floculation, c'est-à-dire l'agglomération des particules sous l'action de la composition chimique de l'eau, se déposent, en partie seulement, dans les réservoirs artificiels. IIs sont véhiculés dans la retenue, vers l'aval, par les courants de densité et constituent un lac de vases et un dépôt dont l'épaisseur croît de haut en bas. Les eaux de surface sont claires. Au moment des grandes erues, il se produit un remous d'underflow. Les dépôts sont très épais contre le barrage et diminuent d'aval en amont. Leur consistance est variable. Ils se solidifient surtout lorsque les éléments sont en majorité siliceux ou silteux, la proportion des collö̈des plus élevée et la granulométrie plus forte. S'il y a dominance d'argiles fines, ces dépôts restent à l'état de boues inconsistantes et sont plus faciles à évacuer lors des vidanges de fond du réservoix [6].

En ce qui concerne les matériaux chargés sur le fond, la valeur de cette partie du débit solide est encore plus difficile à évaluer et jusqu'à présent, le seul procédé efficace consiste simplement à mesurer les apports successifs derrière l'obstacle d'un barrage.

Dans un réservoir, ces matériaux de charriage se disposent à l'amont de la retenue, en formant un delta d'accumulation des dépôts, dont le talus aval évolue en progressant dans la retenue. Le front des dépôts est un talus raide dont l'arête supérieure est au niveau du déversoir, de telle sorte que les dépôts grossiers se font par avancement du talus à front raide et exhaussement au fur et à mesure de l'avancement de ce talus. On constate une bévation du niveau des biefs d'amont par alluvionnement, en même temps que la pente en long de la partie supérieure des dépôts devient moins forte que celle du lit d'origine. On note surtout une variation considérable des apports, d'une crue à l'autre et d'une année à l'autre, en quantités et en proportions granulométriques. Il se produit d'ailleurs un triage des matériaux. 'Très peu d'éléments fins subsistent parmi les matériaux grossiers. Ceux-ci sont pratiquement propres. En général, les matériaux grossiers ne dépassent pas le talus à front raide. C'est pourquoi l'on ne trouve que fort peu de matériaux grossiers a la partie aval des lacs. Cependant, à la faveur des crues, des matériaux. 
grossiers peuvent parfois atteindre la face amont du barrage, où ils sont arrêtés [6].

Quoi qu'il en soit, il est difficile de mesurer la valeur moyenne annuelle des transports solides et d'estimer les parts respectives des matériaux selon leur origine.

Cette difficulté supplémentaire tient d'une part au fait qu'une forte fraction du débit solide s'écoule souvent en quelques jours seulement chaque annee, et d'autre part au fait que ces transports sont irréguliers et se produisent par saccades et reprises partielles. La formation des laves (le phénomène de thixotropie), c'est-à-dire d'un fluide visqueux à forte densité, par mélange d'eau et de matières solides, rend ces phénomènes encore plus complexes. Il se forme, le long d'une rivière, des dépôts temporaires, parfois de longue durée : ce sont les plages de dépôts. En définitive, ce que l'on mesure derrière un ohstacle, tel qu'un barrage de correction torrentielle, est composé à la fois de matériaux solides grossiers et de matériaux fins, susceptibles, au cours d'une reprise (par exemple si le barrage était démoli), d'être transportés en partie en suspension. Il y a enfin une usure souvent rapide des malériaux grossiers qui se fragmentent et produisent des limons. Les matières dissoutes proviennent aussi de matériaux solides qui ont été primitivement charriés sur le fond ou entrainés en suspension sur certaines sections de torrents ou de rivières, pendant un certain temps. Il y a donc une grande confusion des phénomènes.

Il est difficile pour ces raisons de déterminer le résultat final d'ensemble, c'est-à-dire le tonnage ou le volume des matières solides transportés annuellement et s'écoulant en un point déterminé de la rivière considérée; ceci, malgré la minutie et le nombre des mesures qui auraient pu être effectuées dans les petits bassins torrentiels tributaires de la riviere principale. Autrement dit, la somme des transits dans les torrents affiuents en amont d'un grand barrage hydroélectrique et de sa retenue n'a qu'un rapport approximatif avec le transit effectif de la rivière principale, à l'époque considérée.

Le rapport est encore plus variable entre le volume des matériaux solides transportés par la rivière et celui des matériaux qui s'accumulent dans le réservoir.

On peut même dire que le comblement d'une retenue représente chaque fois un cas particulier, en relation évidemment avec l'intensitẻ des transports solides de la rivière principale et de ses affluents, mais en relation également avec les modalités souvent variables du tassement et de la consolidation des boues [7] et particulièrement des argiles [8], de telle sorte que, à l'heure actuelle, et dans l'état exact de nos connaissances en la matière, il n'est pas possible de fixer autre chose que des ordres de grandeur. C'est la conclusion à laquelle on est amené en observant les différences extraordinaires des processus de comblement des retenues. Voici quelques exemples :

Il y a d'abord des retenues, vieilles cependant de plusieurs siècles, qui ne se sont jamais comblées. Le barrage d'Alicante (Espagne) a 300 ans d'existence. Sa retenue est intacte, en raison certainement de la granulométrie de la vase. La consistance des boues étant assez fluide, la simple vidange de fond, régulièrement effectuée, suffit pour les évacuer.

C'est aussi le cas, sans donte, du barrage de Bakhada (Algérie). Cet ouvrage a été construit en 1936. Son réservoir, d'une capacité de 37 millions de $\mathrm{m}^{3}$, est toujours intact, après vingt ans d'existence [6].

D'autres ouvrages, bien que menacés, semblent devoir durer longtemps. C'est le cas de la plupart des barrages des Alpes Françaises, non seulement à cause d'apports relativement modérés, mais aussi en raison des dispositions prises pour l'évacuation d'une partie aussi grande que possible des matériaux transportés. Les exemples suivants le montrent :

Le barrage du Chambon (Isère) sur la Romanche : à la suite d'une vidange récente du lac, on a mesuré des dépôts équivalant à 3 millions de $\mathrm{m}^{3}$, atteignant jusqu'à $15 \mathrm{~m}$ d'épaisseur. Or, la capacité initiale était de 54 millions de $\mathrm{m}^{3}$. Le bassin versant a une superficie de $250 \mathrm{~km}^{2}$ et l'ouvrage est en service depuis vingt-quatre ans. Le comblement se poursuit donc à raison de $500 \mathrm{~m}^{3} / \mathrm{km}^{2} /$ an et atteint globalement, en annéc moyenne, $125.000 \mathrm{~m}^{3}$. Nous ferons observer que ces chiffres représentent seulement le volume des matériaux déposés dans la retenue et non pas la totalité du débit solide. Cela étant, si les conditions restent les mêmes, tant en ce qui concerne le mode d'utilisation des eaux que le nombre ef la nature des purges, la durce de l'ouvrage serail de 4 siècles, éventualité raisonnable.

I e barrage du Sautet (Isère) sur le Drac : nous disposons au sujet de cet ouvrage de renseignements extrêmement complets et intéressants [9]. Cette installation hydroélectrique a été mise en service en 1935. La capacité totale est de $130 \mathrm{mil}-$ lions de $\mathrm{m}^{3}$ et la capacité utile de 100 millions de $\mathrm{m}^{3}$. Le lac de retenue a une superficie de 350 hectares et s'étend sur $8 \mathrm{~km}$ de long. Le bassin versant est de $987 \mathrm{~km}^{2}$ dont 800 font partie du Haut Drac. Les précipitations annuelles sont de l'ordre de $1.450 \mathrm{~mm}$.

Le débit solide total du Drac supérieur a pu être calculé et fixé en moyenne entre 400 et $500.000 \mathrm{~m}^{3}$ par an (charriage et suspension) d'après des mesures précises effectuées à l'amont de la retenue, derriere un ancien barrage, au lieu dit le Motty.

On a pu calculer d'autre part, à l'amont de la 
retenue, le volume du delta sous-lacustre, et estimer de cette manière le débit de charriage, qui s'est trouvé être de $1.331 .000 \mathrm{~m}^{3}$, de 1935 à 1945 , soit $133.000 \mathrm{~m}^{3}$ par an - et à l'aval de la retenue, contre le barrage et au fond du lac, le volume des dépôts de vase, qui a été chiffré, pour la même période, à $700.000 \mathrm{~m}^{3}$, soit $70.000 \mathrm{~m}^{3} \mathrm{par}$ an - ne représentant d'ailleurs que le quart au maximum du transit de matériaux en suspension. Au total, les matériaux de toute origine déposés dans le lac, en année moyenne, représentent un volume de $203.000 \mathrm{~m}^{3}$ correspondant aux $2 / 5$ ou à la moitié du débit solide total. Tout le reste a pu être évacué, soit naturellement, soit à l'aide des chasses d'eau, en utilisant les courants de densité.

On a aussi constaté que depuis la mise en service de l'ouvrage (1935) jusqu'au moment des études (1948), soit en 13 ans, les dépòts de charriage à l'amont de la retenue n'ont réduit le volume de la tranche utile que d'environ 1 à 1,5 million de $\mathrm{m}^{3}$, soit 1 à $1,5 \%$ en 13 ans. Quant aux vases déposées, elles se trouvent dans la tranche morte et les ingénieurs estiment, avec un certain optimisme, qu'elles pourront « sans doute » être en grande partie évacuées à l'aide de l'underflow, par des chasses énergiques. Ce point de vue prête cependant à discussion. De ce qui précède, nous retiendrons à tout le moins cette constatation, que les menaces de comblement dans les barrages des Alpes Francaises sont réelles, mais qu'il existe des moyens de les réduire en partie. La longévité du barrage du Sautet serait, en tout cas, de l'ordre de plusieurs siècles.

Le barrage de Serre-Ponçon (Hautes-Alpes) sur la Durance : les études sont terminées et les travaux de construction de cet ouvrage monumental viennent de commencer. La capacité du réservoir sera de 1.200 millions de $\mathrm{m}^{3}$. Les apports annuels de matériaux solides ont été estimés par diverses méthodes à $805 \mathrm{~m}^{3} / \mathrm{km}^{2} /$ an, soit au total $2.900 .000 \mathrm{~m}^{3}$ dont $300.000 \mathrm{~m}^{3}$ de transports solides sur le fond, 2.00 .000 de $\mathrm{m}^{3}$ de limons et $600.000 \mathrm{~m}^{3}$ de vases.

Ce que nous savons déjà du barrage du Sautet permet de prévoir aussi une longue vie à ce nouveau barrage, car, même en admettant que les $2 / 3$ des matériaux solides soient retenus dans le lac, il faudrait environ six siècles pour le combler. Notons cependant l'incertitude dans laquelle nous sommes sur le pourcentage des matériaux solides qui seront retenus dans le lac. Etant donné l'étendue de ce dernier, il est possible et même probable que les matériaux en suspension qui se déposeront dépassent la proportion observée dans le cas du Sautet. D’autre part, les dépôts du delta sous-lacustre risquent d'entamer plus rapidement qu'au Sautet la tranche utile. Une grande part d'incertitude subsiste.
En Afrique du Nord [6], les constatations ont été beaucoup plus graves:

Le barrage d'Hamis, consiruit en 1879 , puis surélevé, et d'une capacité de 23 millions de $\mathrm{m}^{3}$, était comblé en 1950 à $56 \%$, soit à raison de $200.000 \mathrm{~m}^{3}$ par an.

Le barrage de Ksob, mis en service en 1939 , d'une capacité initiale de 12 millions de $\mathrm{m}^{3}$, était comblé en 1950 à $65 \%$, soit à raison de 700 à $800.000 \mathrm{~m}^{3}$ par an.

Le barrage de Fodda, mis en service en 1932, avait un réservoir beaucoup plus important: 225 millions de $\mathrm{m}^{3}$. En dix-huit ans, son comblement atteint 50 millions de $\mathrm{m}^{3}$, soit plus du cinquième de sa capacité initiale. Dans une soixantaine d'années, si l'on n'y remédie, le comblement sera total et l'ouvrage aura duré... 80 ans!

On peut enfin citer en Afrique du Nord plusieurs retenues dont la capacité initiale était de l'ordre de 10 à 30 millions de $\mathrm{m}^{3}$, comblées maintenant à $100 \%$, dont les installations ne peuvent plus fonctionner qu'au fil de l'cau. Ce sont notamment : les barrages des Cheurfas, de Fergoug, de Saint-Lucien el de Meurad.

D’une manière générale, la sédimentation des retenues hydrauliques dépend des facteurs suivants :

a) Le mode d'exploitation. - Le comblement est plus rapide dans le cas des installations hydroélectriques, car on a le souci de perdre le moins d'eau possible. On l'emmagasine et elle dépose. Les réservoirs destinés à l'irrigation sont moins rapidement comblés, parce qu'on fait un usage plus constant et plus rapide des eaux, ce qui permet d'évacuer la plus grande partie du débit solide en suspension.

b) La configuration des retenues. - Les retenues longues et étroites sont moins vite comblées, parce qu'elles peuvent faire l'objet de vidanges fréquentes et de chasses d'eaux efficaces, assurant un entrainement suffisant des matériaux.

c) La nature des sédiments déposés. - - Si la consolidation des dépòts, qui dépend de la nature chimique et de la grosseur des matériaux, est rapide et volumineuse, il est difficile ensuile de procéder à des purges efticaces.

d) Les facteurs régionaux de la dégradation (sol, relief, pente, climat), c'est-à-dire le taux du débit spécifique solide. Ceci est l'évidence même et il n'est point nécessaire d'insister sur ce point.

A la suite de ces considérations générales, nous examinerons la situation en Yougoslavie et, en particulier, à Iablanica.

\section{LE barrage de IABLANICa}

Cetle installation hydroélectrique, mise en service en 1955 recueille les eaux de la totalité du 
bassin supérieur de la Neretva, dont la superficie est de $2.960 \mathrm{~km}^{2}$. Dans ce total, le périmètre de Iablanica, réduit aux seuls bassins torrentiels débouchant directement dans le lac, occupe un territoire de $667 \mathrm{~km}^{2}$, s'étageant entre 270 et $2100 \mathrm{~m}$ d'altitude et renferme 23 grands torrents, pour la plupart très actifs.

Les forestier's yougoslaves ont concentré tous leurs efforts dans la réalisation d'un vaste programme de défense et de restauration des sols dans ce seul périmètre. Les dépenses relatives aux ouvrages d'art dépassent largement le demimilliard de dinars en cinq ans $\left(^{*}\right)$. Cependant, la cadence des travaux est jugée insuffisante. Le projet général de correction s'élève en effet à 3.500 millions de dinars et sa réalisation est retardée par l'insuffisance des dotations annuelles de crédits.

Les justifications sont pourtant sérieuses : il s'agit, là plus que partout ailleurs, sinon d'arrêter, du moins de freiner, la sédimentation accélérée de la retenue de Tablanica. Celle-ci s'étend sur une longueur de $30 \mathrm{~km}$ et une largeur moyenne de $500 \mathrm{~m}$ en hautes eaux, soit une superficie de $15 \mathrm{~km}^{2}$. La capacité totale est de 318 millions de $\mathrm{m}^{3}$ dont une réserve de comblement - tranche morte - de 18 millions de $\mathrm{m}^{3}$. La contenance utile est donc de 300 millions de $\mathrm{m}^{3}$.

Or, une estimation approximative du débit spécifque solide annuel a été faite à l'aide d'un calcul théorique et par application de la formule de Stiny', modifiée par Hercheulidzé, formule dans laquelle intervient un facteur $m$ appelé « degré de torrentialité $\gg$ et correspondant à un classement des torrents en quatre catégories. Pour le périmètre réduit de Iablanica, le calcul a pu être fait soigneusement, puisque les 23 bassins torrentiels qu'il renferme sont bien connus et que l'on a pu préciser pour chacun d'eux les superficies occupées par chaque nature géologique de terrain. Selon cette estimation, les matériaux transportés annuellement dans le lac auraient un volume moyen de $920.000 \mathrm{~m}^{3}$, ce qui représente $1.515 \mathrm{~m}^{3}$ par $\mathrm{km}^{2}$ de bassin versant. Une estimation moins précise, selon la même méthode, des matériaux provenant de la Neretva supérieure, a également été faite. Elle s'lève à $1.900 .000 \mathrm{~m}^{3}$. Au total, nous pouvons admettre, comme résultat du calcul théorique, le chiffre de 3 millions de $\mathrm{m}^{3}$ de transports annuels, pour un bassin de $2.960 \mathrm{~km}^{2}$, soit $1.000 \mathrm{~m}^{3}$ par $\mathrm{km}^{2} / \mathrm{an}$.

Dans de telles conditions, si tous les matériaux solides se déposaient dans le lac, la retenue de Iablanica serait comblée en un siècle, mais nous savons par divers exemples, et notamment par celui du Sautet, que la moitié au moins, sinon

(*) Le dinar a approximativement la même valeur que le franc français. davantage, des débits solides s'évacue naturellement ou artificiellement à l'aval du barrage. A priori, et sous toutes réserves, le réservoir en cause aurait une durée d'au moins deux siècles. Toutefois, et ceci serait grave, la capacité utile serait entamée très vite, dès la cinquième ou sixième année, puisque la réserve de comblement est seulement de 18 millions de $\mathrm{m}^{3}$.

Ces estimations, toutes théoriques, comportent une grande part d'inconnu. Les observations directes qui auraient beaucoup plus de valeur font à peu près défaut. Ơn n'a procédé à aucune mesure directe du débit solide en suspension et encore moins du charriage sur le fond dans les divers torrents de la région considérée.

Est-il possible, par le simple examen des lieux, lors de la vidange du réservoir, de déterminer avec une certaine précision le volume des matériaux déposés annuellement?

Nous avons eu la bonne fortune de trouver le lac complètement à sec, lors de notre visite, le 29 septembre 1956. Nous avons pu le traverser dans sa largeur et nous faire une idée de l'importance et de la nature de la sédimentation qui s'était opérée au cours de la première année de fonctionnement.

L'épaisseur des vases, dans la section amont du lac, variait de 1 à $10 \mathrm{~cm}$. Elle atteignait $15 \mathrm{~cm}$ à l'embouchure de la Rama. Les dépôts étaient particulièrement épais aux abords de l'ancien lit de la Neretva et augmentaient considérablement d'épaisseur de l'amont vers l'aval. A proximité du barrage, les dépôts étaient malheureusement encore cachés par les eaux. Ces dépôts sont constitués par des couches successives d'argiles noires ou grises, entrecoupées de veines jaunes correspondant à des dépôts plus grossiers, sableux ou calcaires. Chaque crue limoneuse se traduit donc par le dépôt, au fond du lac, de deux couches superposées: une couche inférieure composée d'éléments grossiers de couleur claire et une couche supérieure composée d'éléments fins. Ce sont des « varves $»\left(^{*}\right)$.

L'épaisseur moyenne est difficile à calculer, et il faut espérer que les services hydroélectriques responsables procéderont, s'ils ne l'ont déjà fait, à des relevés précis de profils en travers permettant de chiffrer avec certitude le volume de ces dépôts.

Quoi qu'il en soit, si l'on admet une épaisseur moyenne des dépôts de l'ordre de $0,08 \mathrm{~cm}$ sur

(*) 11 est intéressant de noter que de nombreuses strates, de lordre de 6 à 8 , correspondent à une seule année de dépôts. On sait que de nombreux auteurs utilisent les varves pour dater l'âge des dépôts. Il semble, d'après nos observations, que la méthode serait parfois en défaut, car il n'est pas douteux ici que plusieurs varves représentent une seule année de dépôts, et que le nombre de ces varves ou strates successives peut varier d'une année à la suivante. 
$15 \mathrm{~km}^{2}$ de superficie, cela conduirait au total de $1.200 .000 \mathrm{~m}^{3}$ : volume des boues, c'est-à-dire des matériaux transportés en suspension et déposés dans la retenue, par suite de l'absence de courants. Il faut y ajouter le volume des matériaux charriés sur le fond. Ce volume paraît ici très faible. Il n'y a pas eu encore formation d'un véritable delta à l'amont de la retenue. D'autre part, il n'était pas possible d'estimer de visu la part de ces matériaux, retenue immédiatement à l'amont du barrage, dans la réserve de comblement, car celle-ci était encore sous l'eau. On peut seulement estimer ce volume à $15 \%$ au maximum du volume du débit solide en suspensioin, par analogie avec ce que nous savons par exemple du barrage de Serre-Ponçon, où ce coefficient est estimé, selon les auteurs, à 8 ou $12 \%$. En admettant donc (première hypothèse) le taux de $15 \%$, et en estimant à $50 \%$ la part de matériaux en suspension retenus dans le lac (deuxième hypothèse), le volume des matériaux charriés, entièrement retenus dans le lac, serait de:

$$
\frac{1.200 .000 \times 2 \times 15}{100}
$$

soit $360.000 \mathrm{~m}^{3}$.

Ce bref et très approximatif calcul nous conduit finalement à adopter le chiffre de $1.560 .000 \mathrm{~m}^{3}$, ou 1 million et demi de $\mathrm{m}^{3}$, en chiffres ronds, comme représentant le volume total de matériaux solides accumulés dans le lac, au cours de la première année de fonctionnement du barrage.

II n'est pas superflu de dire combien ce calcul est aléatoire. Nous n'avons pas tenu compte de la configuration de la cuvette, c'est-à-dire de la pente des dépôts. Les surfaces réelles sont plus grandes en effet que celles ramenées à l'horizontale. Les dépôts ont probablement comblé certaines cavités. Bref, le résutat de ce calcul est déjà, de ce fait, fort sujet à caution. Tel qu'il est, et ceci n'est qu'une simple coïncidence, ce résultat n'est pas très éloigné de celui que fournit le calcul théorique, par la formule Stiny-Hercheulidzé, en admettant, ce qui n'est nullement démontré, que les matériaux de charriage ont été retenus en totalité, el les matériaux en suspension à concurrence de $50 \%$. Si ces deux hypothèses étaint exactes, on serait conduit à constater que les apports de matériaux solides de l'année 1955-56 ont été voisins de la moyenne theorique annuelle établie par les formules de Stiny-Hercheulidzé. Il y a encore bien des réserves à faire à ce sujet. C'est seulement après une dizaine d'années d'observations continues que l'on pourra établir les estimations valables, lorsque l'on sera renseigné sur la consistance des vases, l'efficacité des purges, et le pourcentage de matériaux de charriage et de matériaux en suspension retenus dans le lac.
Il est intéressant, ne serait-ce qu'à titre indicatif et malgré l'incertitude de ces diverses estimations, de chercher une justification des dépenses engagées pour la défense et la restauration des sols dans le périmètre de Iablanica.

Le calcul a été fait pour la Durance supérieure, à l'amont du barrage en construction de Serre-Ponçon (10) et il est facile de l'appliquer dans le cas particulier qui nous occupe.

Selon le projet général de restauration du périmètre réduit de Iablanica $\left(660 \mathrm{~km}^{2}\right)$, les dépenses prévues sont les suivantes :

en millions de dinars

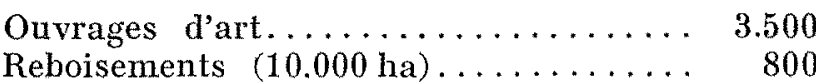

Améliorations forestières

(environ 18.000 ha) 700

Total. ...... $\overline{5.000}$

ce qui représente environ 75.000 dinars à l'hectare. On peut estimer que la réalisation de l'ensemble de ces travaux entraînera une diminution des transports solides de l'ordre de $60 \%$, soit $600.000 \mathrm{~m}^{3} / \mathrm{an}\left(^{*}\right)$ et par conséquent une diminution des dépôts dans le lac, de l'ordre de $300.000 \mathrm{~m}^{3} / \mathrm{an}$.

La durée des travaux étant estimée à 20 ans, la dépense annuelle par $\mathrm{m}^{3}$ de matériaux retenus dans le lac serait de 833 dinars.

Nous ne tenons pas compte des dépenses qui devront être engagées pour l'exécution de travaux agricoles et d'améliorations pastorales. Nous jugeons que ces dépenses sont rentables par ellesmêmes. Bien plus, elles auront une incidence favorable en contribuant pour une part à la diminution de l'érosion superficielle des rersants ce qui, finalement, nous conduit à conclure que la dépense annuelle par $\mathrm{m}^{3}$ de matériaux relenus dans le lac représente environ 700 à 800 dinars au maximum.

Si nous appliquons maintenant ce calcul à l'ensemble du bassin de la Neretva supérieure, où il faudrait aussi intervenir, les résultats seront plus favorables, car ce périmètre élargi est dans un état de dégradation moins avancé que le périmètre réduit de Iablanica. Il n'existe pas ici de projet général de restauration. Il faut done nous contenter d'une estimation générale très approximative. La superficie de ce bassin, déduction faite de celle du périmètre réduit de Iablanica, est de $2.300 \mathrm{~km}^{2}$. A raison de 20.000 dinars de dépenses à l'hectare pour l'ensemble des travaux de construction d'ouvrages d'art et de re-

(*) Ces calcuis sont basés sur l'estimation théorique des matériaux solides transportés, faite à l'aide des formules de Stiny et Hercheulidzé, correspondant d'ailleurs aux dépôts de la campagne 1955-56. 
forestation, la dépense totale à engager serait alors de 4.600 millions de dinars. Les transports solides provenant de cette zone sont estimés à 2 millions de $\mathrm{m}^{3} /$ an el l'exécution des travaux provocuerait en vingt ans une diminution de $60 \%$ des transports solides soit de $1.200 .000 \mathrm{~m}^{3} /$ an et une diminution de $600.000 \mathrm{~m}^{3} /$ an de malériaux retenus dans le lac.

La dépense annuelle par $\mathrm{m}^{3}$ de matériaux relenus serait done de 383 dinars.

Pour lensemble de la Neretva superieure, soit $2.960 \mathrm{~km}^{2}$, la dépense prévisible serait en moyenne de l'ordre de 32.000 dinars à l'hectare et la dépense annuelle par $\mathrm{m}^{3}$ de matériaux relenus par an, grosso modo, de 500 dinars.

Cette dépense est-elle acceptable?

Le même calcul fait pour le hassin supérieur de la Durance, avec une estimation des dépenses de $\mathbf{1 6 . 0 0 0}$ francs seulement à l'hectare, a permis d'estimer la dépense annuelle par $\mathrm{m}^{3}$ de matériaux retenus par an à 154 francs.

Nous donnons cet exemple à titre de compamaison. Il serait maintenant nécessaire de confronter les résultats du calcul effectué pour la Nem retva avec le prix de revient du $\mathrm{m}^{3}$ d'eau emmagasiné dans la retenue de Iablanica. Malheureusement, il ne.nous a pas été possible de recueillir ce renseignement et nous laissons le soin de le faire à nos collègues yougoslaves. Mais nous croyons, étant donné l'ampleur des travaux de construction du grand barrage hydroélectrique de Iablanica, et les dépenses considérables entraînées par le déplacement des voies de communication, que cette confrontation donnerait aisément la justification pleine el entière des travaux entrepris par le Service Forestier de BosnieHerzégovine pour la restauration des sols dans le bassin de la Neretva.

Il faut donc intensifier le reboisement. Tous les auteurs sont d'accord là-dessus. Au Congrès de New Delhi, ce sont non pas des forestiers, mais des ingénieurs hydrologues et électriciens qui ont formulé nettement cet avis. En ce qui concerne par exemple l'Algérie, où le comblement des barrages constitue une menace extrêmement grave, aucun doute ne subsistait dans l'esprit des ingénieurs.

Rappelons cependant que le reboisement a, d'une certaine manière, des conséquences regrettables pour les hydrauliciens : il diminue l'écoulement. Les travaux de reboisement ne trouvent donc leur justification que dans la mesure où ils s'opposent à la dégradation des sols et aux transports des matériaux solides. Ils doivent donc être limités, aux yeux des hydrauliciens, au seul traitement des plaies vives et aux bassins torrentiels les plus actifs. Les forestiers de BosnieHerzégovine ont donc raison de s'attaquer en premier lieu aux terrains les plus dégradés du périmètre réduit de Iablanica. La consolidation des berges dénudées des nombreux torrents de cette zone, à l'aide de clayonnage et de plantations de robiniers, saules, aulnes, etc, portera rapidement ses fruits.

Il faut se rappeler, en eflet, que la plus grande partie des matériaux transportés provient des terrains nus: selon Henin [11], cité par Widmann dans son rapport au congrès de Bled, la dégradation spécifique serait dans le bassin de la Durance supérieure de 15 tonnes $/ \mathrm{km}^{2}$ sous le couvert forestier, et de 45.000 tonnes $/ \mathrm{km}^{2}$, soit 300 fois plus grande, sur les terrains très érodés.

Le reboisement systématique, par contre, n'intéresse pas lhydraulicien, puisqu'il réduit les coefficients d'écoulement.

Cette observation n'est pas seulement valable pour le périmètre de Iablanica. Elle conserve toute sa valeur pour le périmètre de Gardelica, en Serbie, où les reboisements devront s'appliquer d'abord aux surfaces dénudées.

Comme il s'agit dans ce cas particulier d'une érosion superficielle généralisée, il sera nécessaire de faire également appel aux travaux d'amélioration des pelouses, bref de mettre tout en ouvre pour reconstituer un couvert végétal continu de forêts ou d'herbages. Les coefficients d'écoulement n'en seront guère modifiés, étant donné qu'ils sont déjà très faibles en raison de la nature géologique des sols. Mais le débit solide sera diminué d'une manière appréciable.

Sur le Karst enfin, le problème de la sédimentation est infiniment moins préoceupant. Les travaux de reboisement ef d'améliorations pastorales sont justifiés avant tout par des considérations d'équilibre agro-sylyo-pastoral et de meilleure économie rurale.

Rappelons enfin, avant de conclure, que l'on peut envisager pour remédier à la sédimentation des grands barrages, en plus du reboisement, des procédés de lutte passive, à savoir le dévasement par extraction mécanique et dragages, et la construction de barrages de sédimentation, ou débris-bassins.

Les barrages de sédimentation en enrochements ne sont que des palliatifs, des remedes temporaires applicables aux bassins de la Morava du Sud et de la Neretva supérieure.

Il suffit maintenant, pour conclure, de rappeler en les résumant les conclusions du rapporteur M. Widmann et les principales recommandations faites par le Groupe de Travail de la Correction des Torrents, dans le rapport qui a clos la troisième session de cette assemblée tenue en 1956 en Yougoslavie, c'est-à-dire :

En premier lieu, que le gouvernement de Yougoslavie, comme tous les autres gouvernements intéressés, consulte les services qui ont la responsabilité de la conservation des sols avant d'entreprendre de grands travaux d'installations hydroélectriques on de barrages d'irrigation. 
En second lieu, que les Stations de Recherches, avec l'appui des autorités publiques, étudient les problèmes variés et complexes ayant trait à la sédimentation des grands barrages, et notamment :

I. - l'influence des divers types de couvert végétal sur le régime des cours d'eau;

II. - l'importance des transports solides dans les différents types de cours d'eau et, si possible, l'établissement d'une classification des bassins de réception du point de vue de leur sensibilité à l'érosion, et la détermination de méthodes permettant de prévoir approximativement le volume annuel moyen des matériaux de diverses natures transportés par le cours d'eau;

III. - l'efficacité des diverses techniques de correction torrentielle, d'utilisation, de conservation et de restauration des sols;

IV. - le coût de ces travaux et leur rentabilité du point de vue de la lutte contre la sédimentation des réservoirs.

\section{BIBLIOGRAPHIE}

1. G. Labaye. - Note sur le débit solide des cours d'ean. Mémoires et travaux. I-1948.

2. G. Braudeav. - Quelques techniques pour l'étude et la mesure des débits solides. Mémotres et Travanx. I-1951.

3. G. Drovhin. - Sédimentation des réservoirs et problèmes connexes. C.R. Quatrième Congrès des Grands Barrages, New Delhi, 1951.

4. H. Duquennors. - Etude statistique des apports solides et de leur évacuation par courants de densité. C.R. Congrès de New Delhi, 1951.

5. W.C. Cassidx. - The debris problem in the Los Angeles area and its control. C.R. Congrès de New Delhi, 1951.

6. Drovhry, Mallet et Pacouant. - La sédimentation des barrages réservoirs en Algérie. C.R. Congrès de New Delhi, 1951.
7.X. Michon. - Etude du tassement ef de la consolldation des boues. C.R. Troisièmes Journées de l'Hydraulique, Alger, 1954.

8.S. Henn. - Sur quelques propriétés des argiles. Commission pour l'étude de la sédimentation, séance du 25 février 1953.

9. Braudead et Remenmeras. - Quelques observations sur l'alluvionnement dans des réservoirs français. C.R. Congrès de New Delhi, 1951.

10. M. Wromann. - Rapport sur la lutte contre la sédimentation des grands barrages. C.R. Troisieme session du Groupe de Travail F.A.O. de la Correction des Torrents, Bled, 1956.

11. S. Henrn. - Rapport sur l'établissement d'une carte d'érosion des vallées du Haul Drac et de la Haute Durance. Laboratoire des Sols - C.N.R.S., Versailles, 1954.

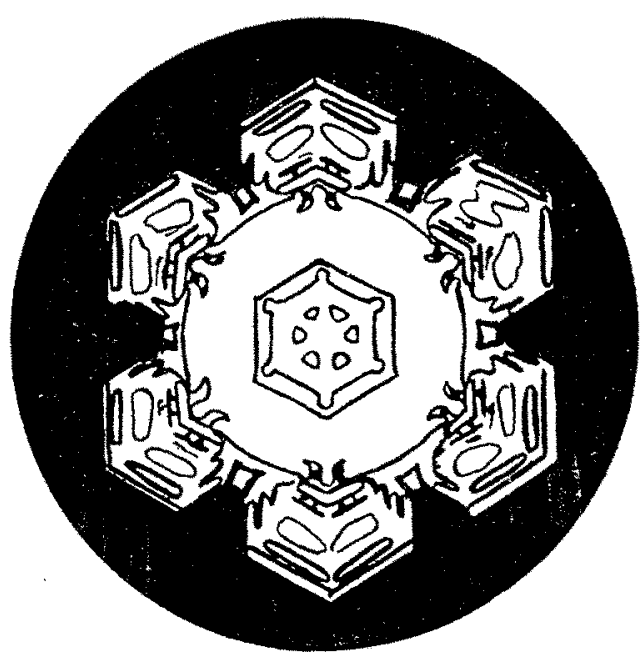

